use has been questioned. ${ }^{4}$ Widening of the retropharyngeal space is suggestive of cellulitis or abscess formation.

In conclusion, we describe a rare case of an 18 month old child with foreign body in the hypopharynx presenting with recurrent respiratory tract infections. Since the ingestion of the foreign body was unnoticed there was a delay in the diagnosis. We stress the importance of increased awareness of foreign body ingestion in children in the A\&E department, particu- larly when the history is unreliable or if the clinical symptoms are atypical.

$1 \mathrm{Lim} \mathrm{CT}$, Loh $\mathrm{KK}$. Ingested foreign body in young children. $\mathcal{F}$ Singapore Paediatr Soc 1992;34:6-10.

2 ConnollyAA, Birchall M, Walsh-Waring GP, Moore-GillonV. Ingested foreign bodies: patient-guided localization is a useful clinical tool. Clin Otolaryngol 1992;17:520-4.

3 Sethi DS, Chew CT. Retropharyngeal abscess - the foreign body connection. Ann Acad Med Singapore 1991;20 581-8.

4 Gautam V, Phillips J, Bowmer H, Reichl M. Foreign body in throat. $\mathcal{F}$ Accid Emerg Med 1994;11:113-5.

\title{
A case of Munchausen syndrome with claims of trauma and haemophilia
}

\author{
Gillian Park, Andrew Huang, Stephen Wright
}

\begin{abstract}
A case of Munchausen syndrome presented with both factitious trauma and factitious haemophilia. He was treated inappropriately with factor VIII concentrate before the history of the presenting complaint could be validated. Clinical suspicion remains the most important aid to diagnosis.
\end{abstract}

(F Accid Emerg Med 1996;13:293-295)

Key terms: Munchausen syndrome; trauma; haemophilia

We report a case of Munchausen syndrome presenting with both factitious trauma and factitious haemophilia.

It is uncommon for cases of Munchausen syndrome to present with trauma as it is very hard to fabricate. Munchausen syndrome presenting with trauma is not the same condition as deliberate self harm but can be as perplexing.

In reply to Asher's original article, ${ }^{1}$ a letter in the Lancet correspondence columns described a casualty presenting with the symptom of haemoptysis which he said began after a lorry crash in Scotland. ${ }^{2}$ A case report ${ }^{3}$ of fabricated trauma 40 years later described the presentation of a man covered in blood who said that a heavy crate had fallen on his chest. The blood came from a facial cavernous haemangioma and the history was increasingly suspicious. In both these cases there was no substantiated trauma.

Factitious haemophilia has been reported in more than one case of Munchausen syndrome. Cases of Munchausen syndrome with false claims of haemophilia have been reported, as have cases of Munchausen syndrome with false claims of haemophilia who then claim to have developed AIDS from contaminated factor VIII transfusion. ${ }^{4}$ There is a case report of a mother with Munchausen by proxy who then developed true Munchausen syndrome and by presenting with psychogenic bleeding duped doctors into treating her for presumed haemophilia and von Willebrand disease. ${ }^{5}$ Our case has been reported more than once with false claims of haemophilia and HIV positivity.

\section{Case report}

A 30 year old man was brought by ambulance to the accident and emergency department of St Mary's Hospital Paddington following liaison with the police. The history from the patient was that one hour earlier he had been riding a bicycle when a car had hit him from behind. He said that he had been propelled forward into a second oncoming car and had catapulted over the handlebars onto the ground. He said that he had not been run over and that both drivers had absconded with their respective vehicles from the scene. The police were called by an unknown person who found the man lying in the road next to his bicycle. No other vehicles involved were seen by the police.

On arrival the cyclist was seen immediately by one of us (AH) who assessed and managed the patient according to ATLS guidelines.

His airway was not at risk and the cervical spine was immobilised. The respiratory rate was $12 / \mathrm{min}$ with $99 \%$ oxygen saturation on 12 litres of oxygen $/ \mathrm{min}$. Auscultation of the chest was unremarkable, with good air entry bilaterally. His pulse rate was 67 beats/min with a blood pressure of $110 / 70$. There was no clinical evidence of external bleeding and peripheral perfusion was normal. He was alert and orientated with equal sized reactive pupils and no focal neurological signs.

From the ATLS primary survey there was no sign of any life threatening trauma but because of the history of the mechanism of injury two large bore intravenous cannulae were inserted 
and $x$ rays were taken of cervical spine, chest, and pelvis. Blood was taken for full blood count, urea, creatinine, electrolytes, glucose, and blood grouping. He was started on two litres of Hartmann solution by intravenous infusion. The $x$ rays showed no evidence of bony injury.

The secondary ATLS survey was performed and revealed the following:

- Abrasions to right anterior thorax

- Abrasions to left elbow

- Right upper quadrant tenderness but a soft abdomen with normal bowel sounds

- Vague right sided pelvic bony tenderness

- Pain on palpating right knee and both ankles but no haemarthrosis nor limitation of range of movement

- Tenderness in the L3/L4 vertebral region on log roll examination.

Multiple old needle marks were noted on the arms and the patient was asked about intravenous drug abuse. He denied this but claimed he was a haemophiliac "using 4000 units of factor VIII with every bleed" and that he was registered with the haematology department of the Royal Free Hospital.

In view of this claim and the finding of abdominal tenderness the surgical team and the haematology laboratory were immediately contacted. Additional blood was sent for clotting profiles and factor VIII assay. There was no evidence for either external or internal bleeding as shown by continuous vital sign monitoring. However 4000 units of factor VIII were given pending surgical assessment.

Further $x$ rays of the skull, lumbar spine, right wrist, knee, and ankle were taken; none revealed bony injury.

While awaiting the factor VIII assay, both the National and the Royal Free Hospital haemophilia registers were contacted - the patient was not known to either register. The police then informed us that the patient had given a false name.

The factor VIII assay was normal at $118 \mathrm{IU} / \mathrm{ml}$.

When challenged about his claim of haemophilia the patient was adamant that there was a positive family history. On requesting his permission for a photograph to be taken he removed his intravenous infusions and left the department. No psychiatric assessment was therefore possible. His incriminating Parthian shot was that both his brother and father were haemophiliacs.

\section{Discussion}

This man falsely claimed to be a haemophiliac. The history of trauma was also a fabrication as there was no corroborating evidence for this. We agree with the statement of Banerjee ${ }^{3}$ that staff in accident and emergency departments need to be aware of trauma as a presentation of Munchausen syndrome. ${ }^{2}$ Further inquiries revealed that this man was known to many hospitals throughout mainland Britain, having attended with numerous aliases. The identifying features included characteristic tattoo markings on his arms. A photograph had been taken by at least one hospital, although he usually made great effort to avoid being photographed.
His activities appear to go back at least six years, when a report in the British fournal of Psychiatry described him as a case of Munchausen syndrome masquerading as AIDS induced depression. ${ }^{6}$

Coincidentally he had previously been encountered by one of us (SW) in another hospital, where he had presented with symptoms of a deep venous thrombosis and a history of HIV and Christmas disease. $\mathrm{He}$ has also presented in Britain and overseas with symptoms of pulmonary embolism, renal colic, acute appendicitis, diabetes, and Kaposi sarcoma (which had since miraculously disappeared). On more than one occasion he has managed to obtain drugs, including opiates, and has abused HIV care groups and support networks, who may unwittingly have leant credence to his claims. The sensitivity which is afforded to genuine HIV cases has also been exploited through direct claims of prejudice to administrative staff. Rather worryingly he appears to have used the name of a genuine AIDS sufferer from one hospital while attending another hospital nearby. He also gives a history of extensive foreign travel, sometimes claiming to be a seaman, and he can speak with various affected accents, including Swedish and American. As in Asher's original paper, the tracing of the migration of this patient leads to a chain of false names with the change in name giving a clue to the original. We agree completely with Cottam et al that this man is the typical adept deceiver with an accurate, albeit erratic, knowledge of his assumed complaint. ${ }^{4}$

The time and resources of the London Ambulance Service, Metropolitan Police, and National Health Service were to no avail in this case. The identifiable cost of radiological investigation and haematology treatment alone was $£ 1382$.

He was treated inappropriately with factor VIII concentrate before the history of the presenting complaint could be validated.

In order to minimise any exacerbation of immune mechanism dysregulation, high purity factor concentrates are currently recommended for HIV positive haemophiliacs, and these preparations are considerably more expensive than the intermediate purity equivalents. A single $4000 \mathrm{IU}$ dose will cost in excess of $£ 1000$.

At present, information on cases of Munchausen syndrome is collected and disseminated by public health consultants, who send letters to regional public health consultants and individual medical directorates in each of the eight regions in the United Kingdom.

A centralised register of cases of Munchausen syndrome has been suggested previously ${ }^{7}$ but raised certain questions. What about confidentiality? How can information be kept up to date? How can information with changing names and aliases be found? We must not forget that a known case of Munchausen syndrome can have organic pathology too. A mislabel of Munchausen could lead to devastating physical and psychological consequences. As was said in $1951^{2}$ we must not forget that those with the Munchausen story, 
though tricksters, may yet be mentally, and sometimes physically, ill; and that we who are not can, as well as being clever, also be kind.

How can cases of Munchausen syndrome be recognised early in the consultation? The clinician's suspicion remains all important.

We thank Mr R Touquet and Dr T Challoner for their advice and assistance in the production of this case report. The tracking of the case by Pamela Dymek is gratefully acknowledged.
1 Asher R. Munchausen's syndrome. Lancet 1951;i:339-41.

2 Birch CA. Munchausen's syndrome. Lancet 1951;i:412.

3 Banajee AK. Trauma and Munchausen's syndrome. Arch Emerg Med 1991;8:217-8.

4 Cottam SN, Cuthbert AC, Parapia LA. Munchausen AIDS and haemophilia. Eur $\mathcal{F}$ Haematol 1991;4b:125.

5 Ayass M, Bussing R, Mehta P. Munchausen's syndrome presenting as haemophilia. Paediatr Haematol Oncol 1993; presenting as

6 Mcdonald J, Wafer K. Munchausen's syndrome masquerading as AIDS induced depression. $\mathrm{Br} \quad \mathcal{F}$ Psychiatry 1989;154:420-1.

7 Jones JR, Horrocks FA. Fictitious epilepsy associated with amnesia. Br F Psychiatry 1987;150:257-8.

\section{Oesophageal perforation: a rare complication of minor blunt trauma}

\author{
Poole Hospital NHS \\ Trust, \\ Longfleet Road, \\ Poole, \\ Dorset BH15 2JB, \\ United Kingdom \\ G L A Cumberbatch \\ M Reichl \\ Correspondence to: \\ G Cumberbatch, Registrar in \\ A\&E Medicine \\ address. \\ Accepted for publication \\ 22 November 1995
}

\section{G L A Cumberbatch, M Reichl}

\begin{abstract}
Oesophageal perforation following blunt trauma is rare and accounts for less than $10 \%$ of all oesophageal ruptures. Review of published reports revealed only two cases of isolated oesophageal perforation after minor blunt trauma, and these were as a direct result of the Heimlich manoeuvre.
\end{abstract}

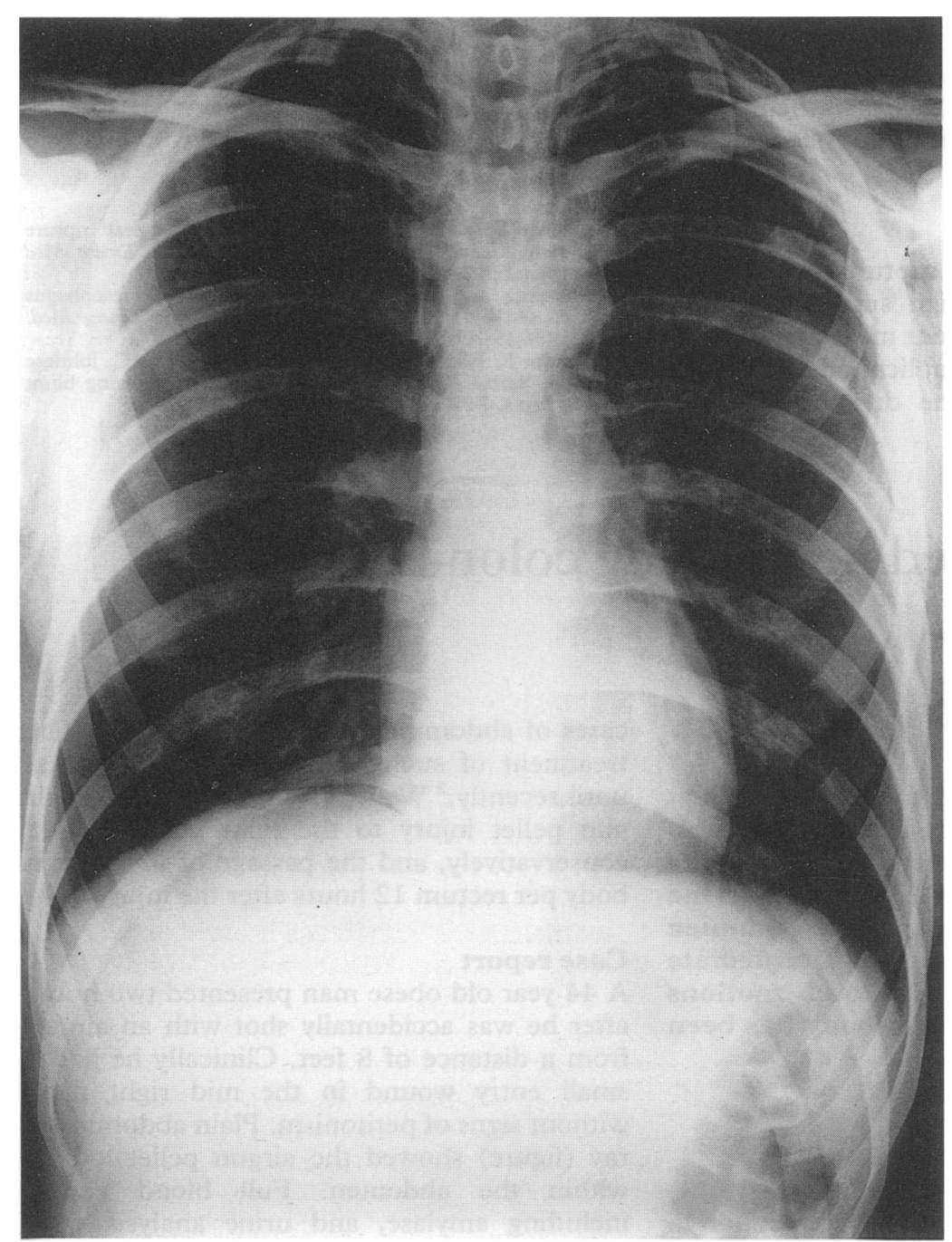

Figure 1 Erect chest $x$ ray with subcutaneous air and a pneumomediastinum.
This paper describes a case of perforation of the oesophagus as an isolated injury following blunt minor trauma.

( f Accid Emerg Med 1996;13:295-296)

Key terms: minor blunt trauma; oesophageal perforation.

Review of published reports revealed only two cases of isolated oesophageal perforation after minor blunt trauma, and these were as a direct result of the Heimlich manoeuvre. ${ }^{12}$ We present a case of perforation of the oesophagus as an isolated injury following blunt minor trauma.

\section{Case report}

A 26 year old man presented to our accident and emergency ( $A \& E$ ) department complaining of neck swelling and dyspnoea, following a blow to the interscapular region of his back during a rugby match. He initially felt "winded" and immediately stopped playing, arranging his own transport to the hospital. On arrival he also complained of dysphagia and a change in his voice.

He had no significant past medical history. On examination he was apyrexial, had a pulse rate of $62 / \mathrm{min}$, a respiratory rate of $24 / \mathrm{min}$, a blood pressure of $139 / 65 \mathrm{~mm} \mathrm{Hg}$, and oxygen saturation of $99 \%$ on air. $\mathrm{He}$ had obvious surgical emphysema extending from the root of his neck down to the nipple line bilaterally. The rest of the physical examination was unremarkable.

Chest radiography (fig 1) revealed marked subcutaneous surgical emphysema, a pneumomediastinum, and a very small apical pneumothorax. Lateral cervical spine $x$ ray (fig 2) showed a large volume of air in the prevertebral space, with anterior displacement of the prevertebral fascia.

A clinical diagnosis of ruptured oesophagus was made. Iohexol (Omnipaque) swallow was arranged urgently and confirmed a perforation in the upper one third of the oesophagus.

He was admitted to the surgical ward and treated conservatively, with intravenous anti- 